\title{
Ein Beitrag zur Lehre von den angeborenen Linsenanomalien.
}

Von

Prof. Schiess-Gemuseus.

Angeborene Formanomalien transparenter Linsen bei sonst gut ausgebildeten Augen gehören zu den ophthalmologischen Raritäten, weshalb ich nicht anstehe, nachfolgenden Fall an dieser Stelle in extenso mitzatheilen:

Ernst S., 12 Jahre alt, aus Rheinfelden zeigte sich am 18. Februar 1885 in unserer Poliklinik und blieb behufs genaverer Untersuchung und Beobachtung bis zum 27. in unserer Klinik. In der Familie finden sich keine besonderen Krankheiten oder Abnormitaten; die Mntter hat als Kind hänfig an Augenkrankheiten gelitten. Der Knabe soll immer schr „kurzsichtig" gewesen sein. Seitdem er die Schule besucht, soll er jeden Winter ejne Augenentzündung bekommen haben, sich aber ausserdem andauernd mit Lesen und Schreiben besehäftigt haben ohne besondere Ermüdung.

Status: Der hochaufgeschossene, magere und etwas blasse Knabe zeigt änsserlich nichts Auffälliges an den Augen. Lidspaiten eher etwas gross; Hornhäute eher gross; vordere Kammer beiderseits ziemlich tief; Irisgewebe von zartem, eher atrophischen Aussehen. Bei der geringsten Bewegung der Augen entsteht in der unteren Hälfte der Iris starkes Schlottern. Linkes 
Auge: Nach Erweiterung der schwarzen Pupille zeigt sich bei Durchleuchtung quer durchs untere Drittel derselben ziehend, eine scharfe, horizontal verlaufende, schwarze Linie, die den unteren Rand der defecten Linse darstellt; der vordere Kapselreflex ist im Uebrigen an seiner normalen Stelle sichtbar and sind keinerlei Locomotionen der Iinse auch bei raschen Bewegungen sichtbar. Der untere Linsenrand ist ganz scharf, so, wie man bei randständiger Iridectomie den normalen Linsenrand sieht, nur anders in seinem Verlauf. Schant der Patient gerade nach vorn, and beleuchtet man das Auge mit dem Spiegel, so schliesst sich an den schwarzen Linsenrand ein nach oben allmählich sich abtönender Schatten aus, der immer breiter wird, je mehr der Patient nach oben schaut.*) Beim Blick des Patienten nach nnten erscheint die ganze Pupille mit Ausschluss der scharfen, schwarzen Linie des Linsenrandes

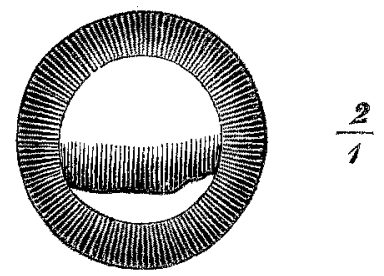

R. Auge.

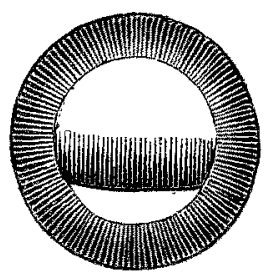

I. Auge.

gleichmässig hell erleuchtet. Die untere Begrenzung des Linsensystems bildet eine horizontale, ganz seicht nach unten convexe Linie, wie die Figur zeigt. Ganz dentlich lassen sich bei schiofer Belenchtung die zarten Zonulafasern verfolgen die sich an den abnormen Linsenrand überall in continuo ansetzen; es sind feine, abwärts verlaufende, parallele Streifchen. Je nachdem man den linsenfreien oder linsenhaltigen Theil der Papillo mar Untersuchung benutzt, bekommt man das aufrechte oder umgekehrte Bild. Die Papille zeigt einen ziemlich bedeutenden atrophischen Bügel; die retinalen Gefässe sind durchschnittlich eng und nehmen einen ungewöhnlich gestreckten Verlauf. Das Pigmentepithel der Chorioidea ist schwach entwickelt und sind die Gefässe derselben sehr deutlich sichtbar.

*) Anf der Figur ist derselbe ungefthr so alggebildet, wie es bei Durchlenchtung mit dem Spiegel beim Blicke gerade nach vorn sich zeigt. 
Rechtes Auge zeigt ein ganz ahnliches Verhalten, nur verläuft die untere Grenzlinie der Linse ganz schwach nach innen zu aufsteigend, um gegen den inneren Linsenrand in zwei scharfen Auskerbungen zum normalen Linsencontour thber zugehen. Auch hier zeigt sich an den schwarzen Grenzcontour anschliessend ein je nach der Augenstellung des Patienten in seiner Breite wechselnder, nach oben allmählich abtönender Schatten. Auch hier sind die feinen, parallelen Zonulafasern, welche Glaskörper und Kammerwasser von einander scheiden, deutlich sichtbar bis zum Linsenrande zu verfolgen. In den hinteren Linsenparthien findet sich hier eine zarte, streifige, in zwei Aeste sich theilende und in einen kleinen runden Knopf endende Linsentrübung; bei gewöhnlicher Tagesbeleuchtung und enger Pupille ist diese Trübung nicht sichtbar. Anch hier kleiner sichelförmiger Bügel.

Die Tension ist beiderseits normal; beiderseits eine måssige allgemeine Beschränkung des Gesichtsfeldes für Weiss; für Roth normal.

S. bei enger Pupille ohne Gläser R. ${ }^{10} / 200$ mit - 12, S. $2 / \pi$. " " " " $" \quad$ L. ${ }^{11 / 200} \operatorname{mit}-16, \mathrm{~S} .1 / 5$. S. bei weiter Pupille ohne Gläser R. ${ }^{15} / 200$ mit +7 , S. ${ }^{2} / 7^{-2} / 5$. $" \quad " \quad, \quad$ " $\quad$, L. ${ }^{15} / 200 \mathrm{mit}+4,5 \mathrm{~S} \cdot{ }^{2} / 7^{2} / 5$. Astigmatismus nicht nachzuweisen. Die Pupillenerweiterung erreicht nur einen mässigen Grad. Patient giebt spontan an, mit weiter Pupille etwas besser zu sehen. Er sieht niemals doppeit; auch führen Versuche, durch Differenzirung des linsenhaltigen und linsenlosen Bildes mit farbigen Gläsern Doppelbilder herbeizuführen, zu keinem Resultat.

In der älteren Literatur finde ich über Formanomalien transparenter Linsen keine Angaben. Auch die Ausbeute in der neueren Literatur ist sehr dürftig. Unter dem Namen Coloboma lentis finden sich wohl eine Anzahl von Einkerbungen des Linsensystems von Heyl*), Dor**) und Anderen erwähnt; sie sind aber, wie die älteren Fälle von Ammon ${ }^{* * *}$ ), Wagner†) und

*) Amnales d'oculistique 1877.

**; Lyon midical 1878.

***) Zeitschrift f. Ophthalmologie. I. Band. \$. 55.

†) Zeitschrift f. Ophthalmologie. III. Band. S. 288. 
Stellwag*) mit Colobomen der Iris und Chorioidea und Retina oder mit Ectopie der Linse verbunden. Nur bei Becker**) finde ich die kurze Angabe: „So fand ich einmal ohne eigentliche Verschiebung der Linse nach oben und aussen die untere, innere Hälfte der Linse durch zwei dicke und breit contourirte, sich unter einem rechten Winkel schneidende, gerade Linien begrenzt." Becker meint, diese Formenänderung sei durch ungleichen Zug der Zonula entstanden. Dann beschreibt Bresgen ${ }^{* *}$ ) ein doppelseitiges :Coloboma lentis; aber beide linsen zeigen perinucleare Trübungen und hintere Corticalistrübungen. Ich habe sehon im Jahre 1871 in Zehender's Klinischen Monatsblättern einen Fall von angeborenem Linsendefect beschrieben and abgebildet. Es handelte sich um ein myopisches in seiner Form sonst normales Auge und war nur eine unbedeutende Trübung des Linsensystems vorhanden; kein Coloboma. Der Fall ist auch Wedl und Bock in ihrem neuesten Werke "Pathologisehe Anatomie des Anges" bei der Zusammenstellung der Formanomalien der Linse entgangen.

Unser Fall ist dadurch ausgezeichnet, dass die linke Linse vollständig klar ist and die rechte nur eine ganz zarte Trübung zeigt, die das Sehvermögen nicht wesentlich herabsetzt.

Ohne Zweifel bandelt es sich um eine angeborene Veränderung, da der Knabe immer als kurzsichtig angesehen worden. Sehr beachtenswerth ist auch die vollständig erhaltene Zonula, die bei genauerer Einstellung und etwas schiefem Einfallen des künstlichen Liehtes sich ausserordentlich schön in ihrer zarten radiären Anordnung verfolgen lissst. - Der eigenthümliche, auf der Figur angedeatete Schatten, der sich an den schwarzen scharfen

*) Stellwag, Ophthalmologie. I. Band. S. 744.

**) Becker, Anatomie der gesunden und kranken Linse. p. 129.

*) A. f. A. IV. 
Randcontour des unteren Linsenbordes anschliesst, sich beim Abwärtssehen ganz verliert, beim Aufwärtssehen immer mehr zunimmt, erklärt sich sehr einfach aus dem Reflex des unteren, fast walzenförmigen Linsenrandès. Es mag dieser starke Reflex auch der Grund sein, warum der Patient, obwohl er bei weiter Pupille mit den entsprechenden Coneavgläsern besser sah, als mit enger Pupille und den corrigirenden Concavgläsern, doch schliesslich die Correction mit Coneavglüsern bei enger Pupille vorzog. Es mag hier nebenbei bemerkt sein, dass der anfungliche Gedanke, den Patienten durch eine künstliche Pupille hindurch den aphakischen Theil derselben benutzen zu lassen, fallen gelassen wurde.

Da bei unserem Falle die Zonula in völliger Continuität vorhanden ist, bin ich eher geneigt für das Zustandekommen einer solchen abnormen Configuration, resp. das Ausfallen eines Theils des Linsenparenchyms die Erklärung von Heyl anzunehmen, dass einzelne Aeste der Arteria hyaloidea mangelhaft entwickelt waren oder ganz gefehlt haben. Die Beeker'sche Anșicht eines verschiedenen Zuges der Zonula ist wohl nur auf ektopirte Linsen oder solche, bei denen an einer Stelle die Zonula fehlt, anwendbar. Immerhin müssen wir annehmen, dass das spätere Leben der Linse nicht gelitten, sondern dass sie von den Randtheilen her gerade so weiter wachse, wie ein ganz wohl geformtes Linsensystem, da auch rechts, wo wir ja eine feine Linsentrübung haben, dieselbe den unteren Linsenrand völlig frei kässt. - Von anderweitiger mangelhafter Entwickelung in den Augen unseres Patienten wäre allenfalls noch die dentlich atrophische Bildung der. Iris, die flacher und in ihrem Balkensystem wenig entwickelt erscheint, auch auf Mydriatica weniger reagirt, als normale Individuen gleichen Alters, anzuführen.

Zum Schlusse mache ich noch darauf aufmerksam, dass in jenem früheren Falle und hier es sich um myopische 
Augen handelt; hier sogar um höchste Grade der Myopie. Diejenigen, welche die hohen Grade der Myopie mit ihren Bügeln mit mangelhaftem Abschluss der hinteren unteren Bulbuswandungen in Zusammenhang bringen, finden hier vielleicht eine Brücke zur mangelhaften Bildung der Linse. Ich für meinen Theil begnüge mich, auf dieses Nebeneinanderbestehen aufmerksam gemacht $z a$ haben. 